\title{
LV-PP-1-6
}

\section{Clinical analysis of the outcomes after receiving a liver graft that abandoned transplantation due to poor graft conditions in the centers allocated as a priority}

\author{
Ho Joong CHOI*, Changho SEO, Sung Eun PARK, Joseph AHN, Tae Ho HONG, Young Kyoung YOU
}

Department of Surgery, Seoul St. Mary's Hospital, The Catholic University of Korea, Seoul, Korea

Introduction: Depending on the recipient's condition and lack of donors, even liver grafts with poor conditions may need to be transplanted. This study was conducted to analyze the outcomes after receiving a liver graft that abandoned transplantation due to poor graft conditions at the preceding centers.

Methods: From January 2010 to September 2020, deceased-donor liver transplantation (DDLT) was performed in 161 patients in our center. Among them, 127 patients (allocated group) were preferentially allocated to our center by KONOS and the remaining 34 patients (abandoned group) received liver grafts that were abandoned by other transplant centers due to poor organ conditions. Various perioperative factors and postoperative outcomes were compared.

Results: There was no difference in recipient factors before transplantation, and the donor had a longer stay in the ICU in the abandoned group. The operation time was less in the abandoned group, but there was no statistical difference $(p=0.06)$, and there was no difference in ischemic time or transfusion between the two groups. Postoperative ICU hospital stay was longer in the abandoned group $(p=0.04)$, but postoperative in-hospital mortality was not different between the two groups. There was no difference between the two groups in long term survival after transplantation.

Conclusions: Even if the graft that was abandoned due to poor condition, good results can be obtained if the transplant is carried out according to the recipient state. And as a result, it is expected that the discarded graft can be reduced. 